\title{
Elementary Proof of Existence of a Subordinate Smooth Partition of Unity on Smooth Manifolds
}

\author{
Yu-Lin Chou*
}

\begin{abstract}
We give in particular an elementary proof of the existence of a smooth partition of unity subordinate to any given open cover for smooth manifolds. As a side note, given are also two elementary proofs of the existence of a subordinate partition of unity for topological manifolds. These in particular fill a gap in the related literature.
\end{abstract}

MSC 2020: 54-01; 57N99

Keywords: partitions of unity; smooth manifolds; topological manifolds

Throughout, a partition of unity is always assumed to be associated at least with continuity.

Although the concerned result is well-known, as far as the author is aware, there seems no recorded elementary proof of the existence of a smooth partition of unity subordinate to any given open cover of a given smooth manifold. On the other hand, an elementary proof for compact smooth manifolds is readily available (e.g. Tu [3]). By an elementary proof we mean a "widely accessible" short proof depending at most on regular textbook results 1

We wish to show that there is an elementary way to transfer the proof idea for the compact case to the general case.

We will prove for spaces slightly more general than smooth manifolds. A secondcountable Hausdorff space is called smooth locally Euclidean if and only if for every

*Institute of Statistics, National Tsing Hua University, Hsinchu 30013, Taiwan, R.O.C.; Email: y.l.chou@gapp.nthu.edu.tw

\footnotetext{
${ }^{1}$ Some "philosophical" comments regarding the position of an elementary proof might be in order. The position of an elementary proof in particular always includes being a potential source to deepen the understanding of the "problem" at hand. Selberg's elementary proof (Selberg [2]) of the prime number theorem is certainly a classic example. Here the fact that the paper contributes to winning a prize is not essential; like Feynman once uttered, the prize really is the pleasure of finding things out as well as the gained understanding.
} 
point of it there are some neighborhood of the point and some $n \in \mathbb{N}$ such that the neighborhood is homeomorphic to $\mathbb{R}^{n}$ and the coordinate open cover thus obtained forms a smooth atlas in the evident sense.

We should like to prove

Theorem 1. If $M$ is a smooth locally Euclidean space, and if $\left\{G_{\theta}\right\}_{\theta \in \Theta}$ is an open cover of $M$, then there is some smooth partition of unity subordinate to $\left\{G_{\theta}\right\}$.

Proof. Given any point of $\mathbb{R}^{n}$ and any neighborhood of the point, it is well-known that there is some smooth function $f: \mathbb{R}^{n} \rightarrow[0,+\infty[$ (compactly) supported in the given neighborhood such that the co-zero-set $\left\{x \in \mathbb{R}^{n} \mid f(x) \neq 0\right\}$ of $f$ contains the given point; such a function is immediately adaptable from the usual choice

$$
x \mapsto \exp \left(\frac{1}{|x|^{2}-1}\right) \mathbb{1}_{B_{n}(0,1)}(x)
$$

on $\mathbb{R}^{n}$. Here $\mathbb{1}_{B_{n}(0,1)}$ denotes the characteristic function of the unit open ball $B_{n}(0,1)$ in $\mathbb{R}^{n}$.

Given any $\xi \in M$ and any neighborhood $G$ of $\xi$, let $U$ be the intersection of $G$ and some coordinate neighborhood of $\xi$. If $\varphi$ is a coordinate map on $U$, the $\varphi$-image $\varphi^{1)}(U)$ of $U$ is a neighborhood of $\varphi(\xi)$; choose a smooth function $f: \mathbb{R}^{n} \rightarrow[0,+\infty[$ supported in $\varphi^{1)}(U)$ with co-zero-set containing $\varphi(\xi)$. Since $\varphi$ is a diffeomorphism, in particular the commutativity of closure operation and the preimage map $\varphi^{-1)}$ implies that $f \circ \varphi: U \rightarrow[0,+\infty[$ is a smooth function supported in $G$ with co-zero-set containing $\xi$. Then the function $g: M \rightarrow\left[0,+\infty\left[\right.\right.$ defined by $\left.g\right|_{U}:=f \circ \varphi$ and $\left.g\right|_{U^{c}}:=0$ is a smooth function on $M$ supported in $G$ with co-zero-set containing $\xi$.

Since for every $\xi \in M$ there is by assumption some $\theta \in \Theta$ such that $\xi \in G_{\theta}$, for every $\xi \in M$ we choose a smooth function $g_{\xi}: M \rightarrow\left[0,+\infty\right.$ [ supported in $G_{\theta}$ with co-zero-set containing $\xi$, and let $V_{\xi}$ be the co-zero-set of $g_{\xi}$.

Upon considering the Alexandroff (one-point) compactification $M_{\infty} \equiv M \cup\{\infty\}$ for $M$ (possibly without denseness) and choosing a co-compact set $A$ in $M$, we obtain an open cover $\{A \cup\{\infty\}\} \cup\left\{V_{\xi}\right\}_{\xi \in M}$ of $M_{\infty}$; this is possible as the given topology of $M$ is by construction the relative topology of $M$ in $M_{\infty}$. Since $M_{\infty}$ is compact by construction, fix finitely many $\xi_{1}, \ldots, \xi_{m} \in M$ such that $V_{\xi_{1}}, \ldots, V_{\xi_{m}}, A \cup\{\infty\}$ form an open cover of $M_{\infty}$.

Since every point of $M$ lies in some $V_{\xi_{i}}$, the proof then completes itself by the standard procedure: As the functions $g_{\xi_{i}} / \sum_{i=1}^{m} g_{\xi_{i}}(i=1, \ldots, m)$ form a smooth partition of unity not necessarily subordinate to $\left\{G_{\theta}\right\}$, and as for every $i=1, \ldots, m$ we can by construction choose exactly one $I(i) \in \Theta$ such that $g_{\xi_{i}}$ is supported in $G_{I(i)}$ and $\xi_{i} \in V_{\xi_{i}}$, 
a re-indexing by defining

$$
h_{\theta}:= \begin{cases}\sum_{\substack{1 \leq i \leq m ; \\ I(i)=\theta}} \frac{g_{\xi_{i}}}{\sum_{i=1}^{m} g_{\xi_{i}}}, & \text { if } I(i)=\theta \text { for some } i ; \\ 0, & \text { if } I(i) \neq \theta \text { for all } i\end{cases}
$$

for all $\theta \in \Theta$ gives a smooth partition of unity on $M$ subordinate to the given open cover $\left\{G_{\theta}\right\}_{\theta \in \Theta}$.

We now turn to topological manifolds. Although a standard proof of the existence of a partition of unity subordinate to any given open cover of a given compact topological manifold is elementary, for general topological manifolds an elementary proof seems not easy to locate in the literature. For instance, the argument considered in Munkres [1] would not be elementary with respect to the definition we agreed early on. We thus intend to fill this gap.

We will again consider spaces slightly more general than topological manifolds. A second-countable Hausdorff space is called locally Euclidean if and only if for every point of it there are some neighborhood of the point and some $n \in \mathbb{N}$ such that the neighborhood is homeomorphic to $\mathbb{R}^{n}$.

We give two elementary proofs of the following

Theorem 2. If $M$ is a locally Euclidean space, then for every open cover of $M$ there is some partition of unity subordinate to the cover.

Proof. Since $M$ is in addition from assumption a locally compact Hausdorff space, the space $M$ is a regular space. But $M$ is also second-countable by definition, it then follows from the Urysohn metrization that $M$ is metrizable. The metrizability of $M$ implies that $M$ is paracompact, which implies the desired existence by a well-known textbook result. This gives the first proof.

As every smooth real-valued function on a smooth manifold is continuous, the second proof follows immediately from the proof of Theorem 1, with verbal changes only.

\section{References}

[1] J.R. Munkres, Elementary Differential Topology, Princeton University Press, 1963.

[2] A. Selberg, An elementary proof of the prime-number theorem, Annals of Mathematics 50 (1949) 305-313.

[3] L.W. Tu, An Introduction to Manifolds, Springer, 2011. 